\title{
Implementing the six sigma model on the treatment of congenital hypothyroidism: An analytical hierarchy process
}

\author{
Hosein Ebrahimipour ${ }^{1}$, Salman Shojaei ${ }^{1} * \mathbb{D}$, Ameneh Esfandyari $^{2}$, Salah Eddin Karimi ${ }^{3}$, Ali Vafaee Najar ${ }^{1}$, \\ Habibollah Esmaily ${ }^{1}$
}

Received: 4 Dec 2020

Published: 21 Sep 2021

\section{Abstract}

Background: Congenital hypothyroidism is a disease able to cause severe mental retardation and developmental delays. However, timely diagnosis and treatment of infants with this disease could prevent relevant complications. This study aims to investigate the effects of the implementation of the Six Sigma model on reducing the treatment initiation time in infants with congenital hypothyroidism in the population chosen from Samen Health Center in Mashhad.

Methods: In this quasi-experimental study, the referral process of infants for congenital hypothyroidism screening and treatment was evaluated for the time period starting from March 20, 2017, to March 19, 2018, using the standard five-phase quality strategy, description, measurement, analysis, improvement and control phase (DMAIC), based on Six Sigma. Data were collected using the sampling form of the national screening program for congenital hypothyroidism. To analyze the data, software including Expert Choice V11, Microsoft Excel 2013, and SPSS 18, were utilized. In addition, a p-value less than 0.05 was considered statistically significant.

Results: The number of infants who entered the intervention process was 4,574, of whom 51.3\% (2346 infants) were boys. The mean time to start treatment before the implementation of the model was $21.72 \pm 7.72$ days, which decreased to $17.41 \pm 6.47$ days after the implementation of the model $(\mathrm{p} \leq 0.05)$. Besides, $81.8 \%$ of the patients received treatment during infancy before the intervention, which increased to $94.1 \%$ after it. After the implementation of the Six Sigma model, the Sigma level of treatment initiation improved from 2.41 to 3.06 .

Conclusion: Six Sigma could be used as an intervention tool for improving indices of health intervention processes.

Keywords: Congenital Hypothyroidism, Mental Retardation, Six Sigma, DMAIC, Sigma Level, Analytical Hierarchy Process

Conflicts of Interest: None declared

Funding: None

\section{*This work has been published under CC BY-NC-SA 1.0 license.}

Copyright $\odot$ Iran University of Medical Sciences

Cite this article as: Ebrahimipour H, Shojaei S, Esfandyari A, Karimi SE, Vafaee Najar A, Esmaily H. Implementing the six sigma model on the treatment of congenital hypothyroidism: An analytical hierarchy process. Med J Islam Repub Iran. 2021 (21 Sep);35:121. https://doi.org/10.47176/mjiri.35.121

\section{Introduction}

Congenital hypothyroidism $(\mathrm{CH})$ is one of the major preventable causes of mental retardation in newborns. Disorders of important organs, such as the central nervous and skeletal systems, occur in the presence of hypothyroidism in the fetus $(1,2)$. Since hypothyroidism symp-

Corresponding author: Salman Shojaei, shojaeis1@mums.ac.ir

1. Social Determinants of Health Research Center, Mashhad University of Medical Sciences, Mashhad, Iran

2. Samen Health Center, Mashhad University of Medical Sciences, Mashhad, Iran

3. Social Determinants of Health Research Center, Health Management and Safety Promotion Research Institute, Tabriz University of Medical Sciences, Tabriz, Iran toms appear gradually, the diagnosis is usually delayed. Clinical symptoms increase over time, mental and physical retardations develop during the following months, and the clinical picture of the disease is complete at the age of 3 to 6 months (3). Hypothyroidism often has few symp-

$\uparrow$ What is "already known" in this topic:

Timely screening, diagnosis and treatment, of $\mathrm{CH}$ reduces the complications of the disease. Reducing the IQ of untreated patients has high costs for the family and the community.

\section{$\rightarrow$ What this article adds:}

The Six Sigma model was used for the first time to reduce treatment delays in neonates with congenital hypothyroidism. The use of this model was effective and can be used in other studies. 
toms, and only about $5 \%$ of cases have clinical symptoms during the early days after birth. Accordingly, based on clinical findings, only $10 \%$ of infants in the first month, $35 \%$ in the first quarter, $70 \%$ in the first year, and $100 \%$ within 3-4 years after birth are diagnosed with hypothyroidism (4). Numerous studies have revealed that 10 to $15 \%$ of patients with $\mathrm{CH}$ have intelligence quotient disorders with learning difficulties at school (5).

The total incidence of this disease in the world is estimated to be 1 in 3,000 to 4,000 live births (6). According to studies conducted in Iran, the prevalence of this disease varies within the range of 1 in 370 to 1 in 1000 individuals in the country. The reports of the program in Iran from 2005 to September 2010 showed that the prevalence of the disease was 1 in 670 live births (2). According to a study conducted in the United States and France, the costbenefit ratio of screening was estimated to be 10 to 1 and 12 to 1 , respectively (7). In the Iranian national program for 'neonatal screening', the best time for sampling and treatment initiation was determined to be 3-5 days after birth and during the neonatal period (until 28 days after birth), respectively (2). A study on the treatment of patients with hypothyroidism showed that patients treated in the first 20 days after birth had a higher average IQ score than those treated later (8). According to the inverse relationship between the age of diagnosis and treatment of patients and the decrease in IQ of patients with an average of 89 to 54 points, based on studies conducted in Sweden, the importance of screening and timely treatment of patients is clearly defined (9).

Quality improvement models in health services provide safe, timely, efficient, and effective services. One of the models of quality improvement in healthcare is the 6 Sigma model (10).

Six Sigma is a systematic and statistical approach aimed at specifying defects of processes and reducing errors in business and clinical processes gone through by spending a long time and high costs with poor outcomes. Six Sigma has been introduced as a systematic and powerful approach to achieving improvements in the quality of health and treatment services as well as cost control (11).

Healthcare research shows that Six Sigma is able to reduce the length of stay (LOS) among patients, bed turnover time (12-14), costs $(12,14,15)$, and mortality (12). In addition, it enhances the use of resources $(15,16)$, patient satisfaction (15), as well as performance indices of the operating room (12).According to the results of similar research and given the importance of reducing screening and initiation delays in treating infants with congenital hypothyroidism, this study was conducted to reduce treatment initiation delays in the population selected from Samen Health Center located around Razavi holy shrine, Mashhad. The mentioned center is one of the five centers in Mashhad, with a population of 98,000. About 30 million pilgrims travel to this city every year. In addition, seven urban health care centers with 20 health posts, 12 hospitals, about 500 doctors' offices, infirmaries, clinics, and private laboratories are covered and supervised by this center. The results of this study could be effective in reducing the screening time, initiation time of treating iden- tified patients as well as consequences of delayed treatment among patients such as growth and development problems, thereby decreasing the burden of the disease.

\section{Methods}

In this quasi-experimental study, all infants born from March 20, 2017, to March 19, 2018 (4,574 cases), who referred to hospitals, urban health centers, and health posts affiliated to Samen Health Center of Mashhad were screened. Infants who had moved to another area or had died during the study period were excluded. The reason for selecting this center was the variety of sampling units, including government, charity and private. Also, the native population and other nationalities resided in this area. In addition, data were collected using the standard sampling form of the national screening program for congenital hypothyroidism. The intervention was performed based on the five steps of Six Sigma DMAIC (17) as follows:

First step (description phase): At this stage, the process of discharging newborn infants from hospitals until treatment initiation, in case they were ill, was drawn with the participation of process owners at urban health centers, health posts, hospitals, and refierence laboratories, and the process was observed. Next, the drawn process was confirmed by the process owners Figure 1.

Second step (measurement phase): At this stage, the duration of the whole process and each of the stages of the process were determined. For this purpose, times recorded in the screening forms, the registration portal of samples taken, and treatment records of the patients were extracted, using which the Sigma level, mean, and standard deviation of the processing time were separately calculated by the sampling time, test response time, and treatment initiation time.

Third step (analysis phase): At this stage, the causes of the problem (delays in initiating the treatment) were determined by drawing the Ishikawa diagram. The brainstorming session was attended by sample collectors, sample carriers (people in charge of the collection, quality control, and dispatching of the samples to the central laboratory), central laboratory staff, program focal points (a pediatric specialist serving as a university faculty member, a consultant physician, and experts of treating patients with congenital hypothyroidism), and disease experts (individuals with their education and activities being in the field of diseases). Next, a solution was produced for each of the problems through brainstorming. To formulate final proposals, seven experts with at least three years of experience in the relevant field were asked about the importance of each item using the five-point Likert scale, which ranged from score 1 for low importance to score 5 for highly important. Accordingly, proposals with an average score of above 4 were included in the study. In the end, nine proposals were selected for the intervention. Besides, the AHP technique was used to determine and prioritize the intervention. Four criteria, including effectiveness, practicality, cost efficiency, and shortness of the intervention time, were regarded as criteria for comparing proposals. Four people were selected as voters, and the Fundamental scale of Saaty was used in comparative scor- 


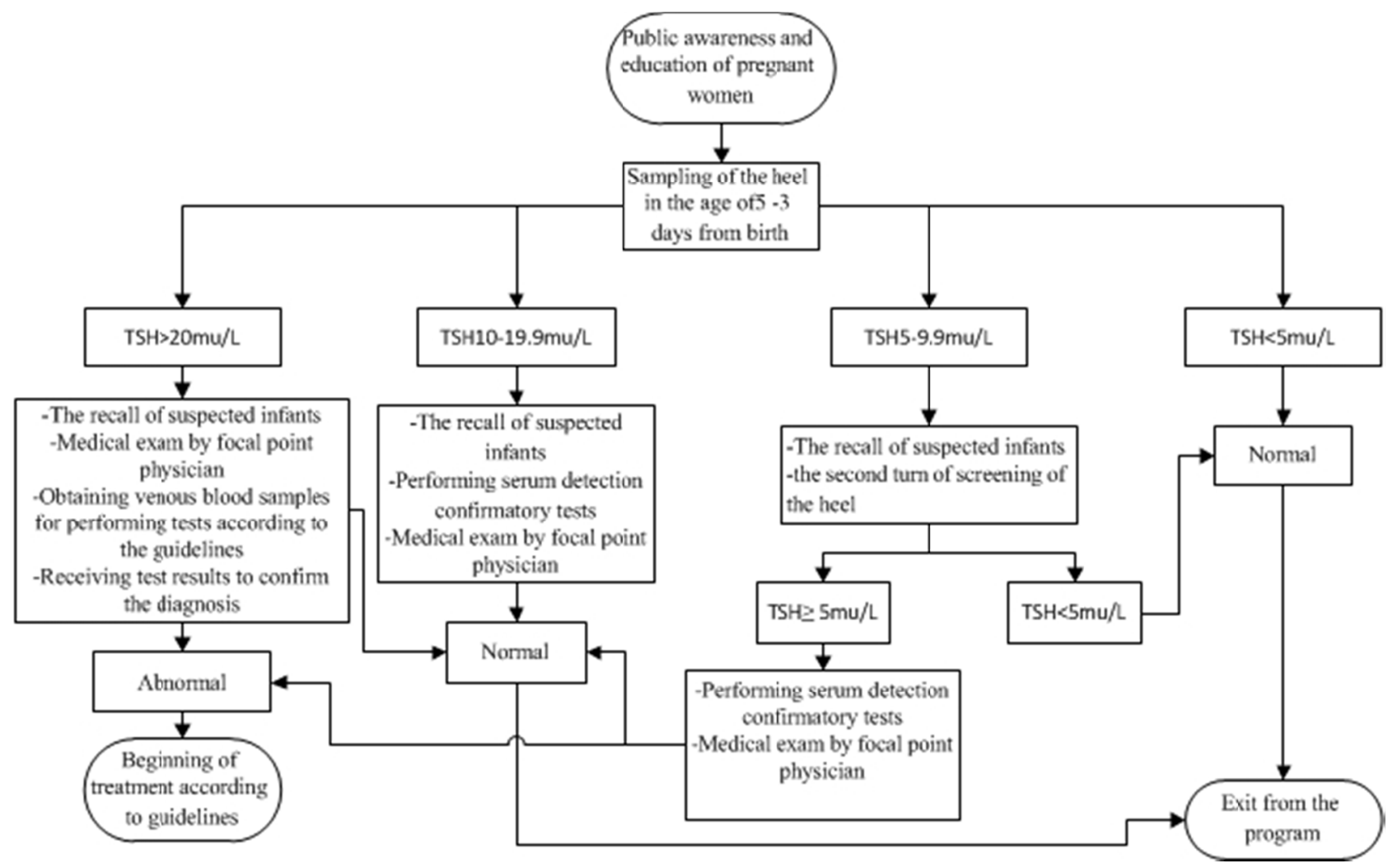

Fig. 1. Process of screening of infants and treatment of patients with congenital hypothyroidism

ing as a standard scale to determine the weight of each of the 9 options (18).

The number on the diameter of the matrix was 1 , which indicated the same importance of each option compared to itself. After collecting the questionnaires, the incompatibility rate of the comparisons was determined as follows:

First of all, the weighted sum vector (WSV) was calculated by multiplying the paired comparison matrix (D) by the relative weight vector as follows:

$$
W S V=D . W
$$

Next, WSV elements were divided by the relative weight vector, which is called the compatibility vector (CV).

Additionally, the largest specific value of the paired comparison matrix $(\lambda \max )$ was calculated. To calculate $\lambda \max$, the mean of compatibility vector elements was calculated. In addition, the incompatibility index was calculated as follows:

$$
\text { C.I. }=\frac{\lambda_{\max }-n}{n-1}
$$

To calculate the incompatibility rate (IR), the following formula was used:

$$
\text { C.R. }=\frac{C . I .}{R . I .}
$$

Next, the random inconsistency index (I.R.I) was extracted from the random inconsistency table that was based on the simulation.

After making sure that the incompatibility index was acceptable (less than 0.1) following comparing the pair and given the higher weight of the comments of one of the members, we calculated its geometric mean for combining judgments based on the following formula:

$$
\bar{a}_{i j}=\left(\prod_{k=1}^{l} a_{i j}{ }^{w_{k}}\right)^{1 / N}, \quad N=\sum_{k=1}^{l} w_{k}
$$

To prioritize the options, the tables were normalized according to the following formula:

$$
r_{i j}=\frac{\bar{a}_{i j}}{\sum_{i=1}^{m} \bar{a}_{i j}}
$$

At the final stage of the hierarchical analysis, to determine the best options in order of priority, normalized values of the normalized paired comparison table were multiplied by the corresponding mean values of the criteria, and the final table was obtained (18). Expert Choice V11 was used to do all calculations.

Fourth step (improvement phase): In order to follow the designed steps, an action plan was developed. In addition, the required steps for each specified intervention, as well as the person in charge of the steps, were specified. Next, the program was notified by the head of the health center, and its progress was assessed based on the determined indices during a three-month period.

Fifth step (control phase): At this stage, i.e., one year after starting the intervention, the mean times of receiving the first and second samples, test results, and treatment initiation were calculated once more to determine the intervention impact, according to the steps of the first stage. Data analysis was performed by a paired sample t-test in 
SPSS 18.0. In addition, p-values less than 0.05 were considered significant.

Results

The results of this study are presented in three sections as follows:

Determining Process Problems: The following diagram was drawn after determining the sampling process for treating patients. After brainstorming, the major and minor causes of treatment delays were extracted by nine people from among the owners of the process, with its fishbone diagram drawn in Figure 2.

Determining intervention priorities: Initially, the hier-

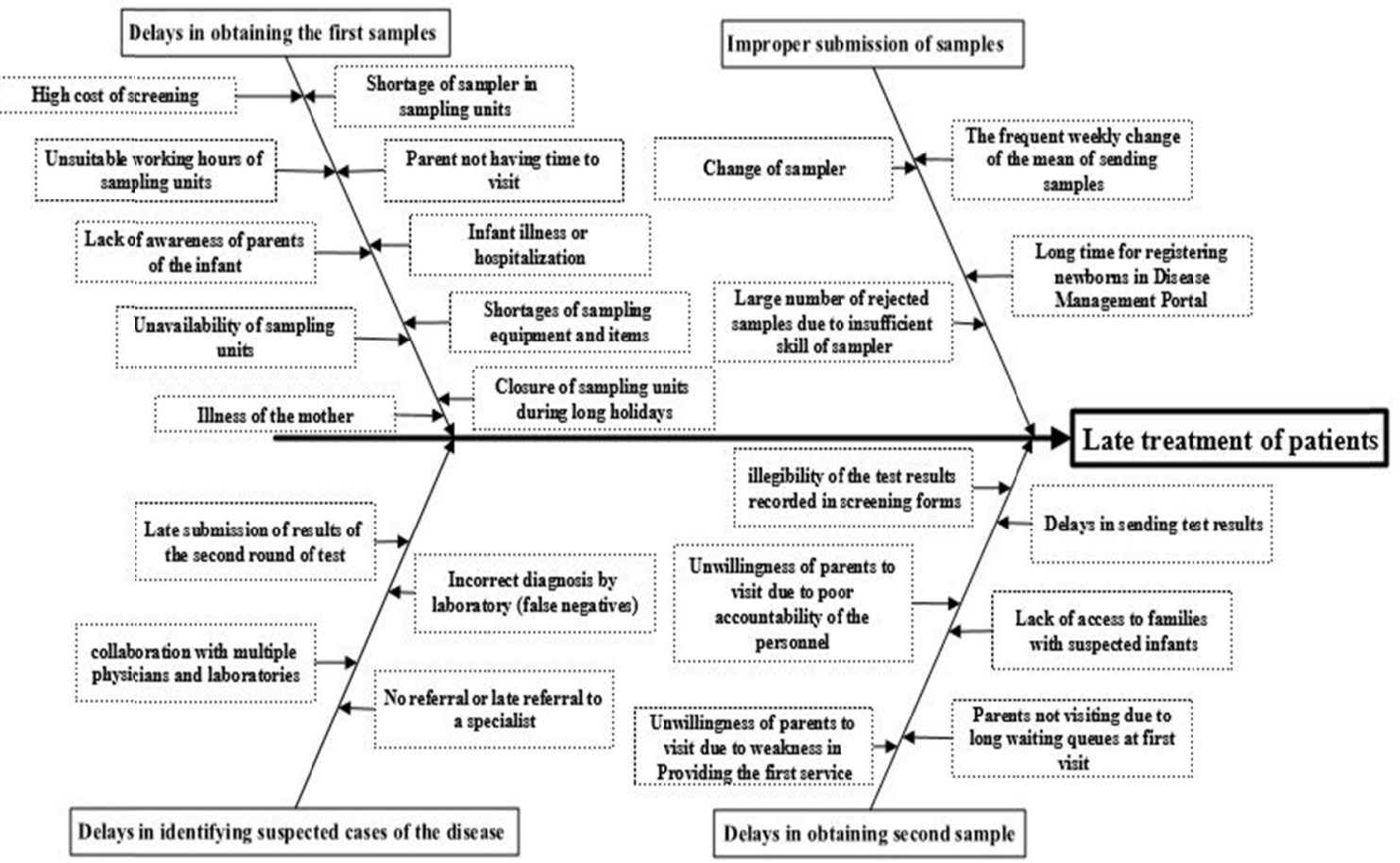

Fig. 2. Fishbone diagram of delayed treatment of patients with congenital hypothyroidism

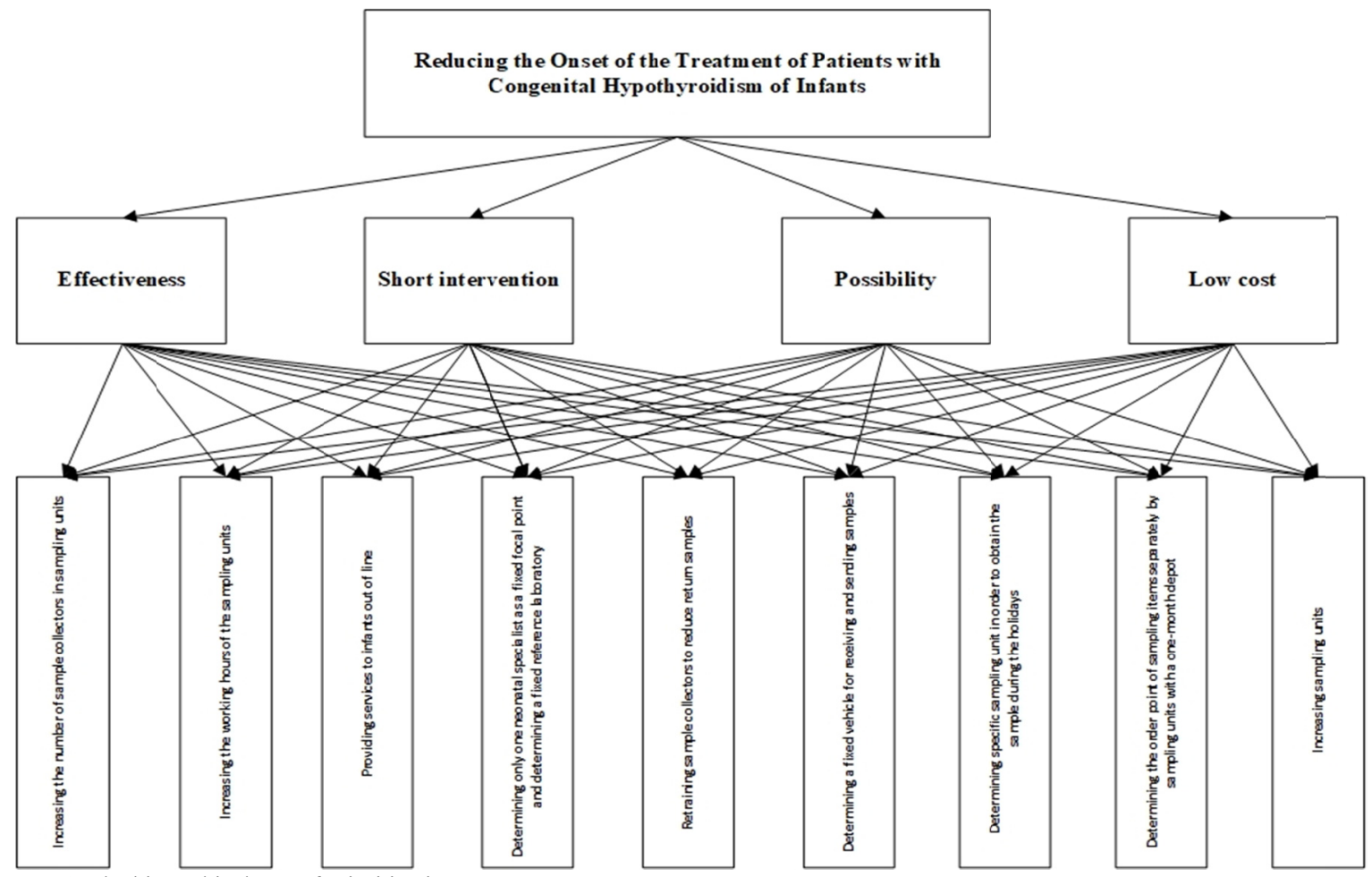

Fig. 3. The hierarchical tree of prioritization 
archical prioritization tree of evaluation indices was drawn as follows in the next part, Figure 3.

Next, the options selected by the members were compared in pairs, and the following Table 1 was produced by applying the importance of the members' opinions (normalization).

After the pairwise comparison of the importance of the criteria and considering the weights of the members' comments, the following Table 2 was obtained.

After the calculation, the final Table 3 was produced by applying coefficients of the criteria in the options.
According to the table above, the three options with the highest weight were prioritized for the intervention.

Intervention results: The total number of the screened infants was 4,574, of whom 2,346 were boys. According to Table 4, the mean time of the first sampling task decreased from 4.68 days before the intervention to 4.39 days after the intervention. In addition, the mean time of the second sampling task decreased from 18.97 days (before the intervention) to 13.52 days (after the intervention); similarly, the time interval between the sampling task and treatment initiation decreased from 21.72 days

Table 1. Pair comparison of items

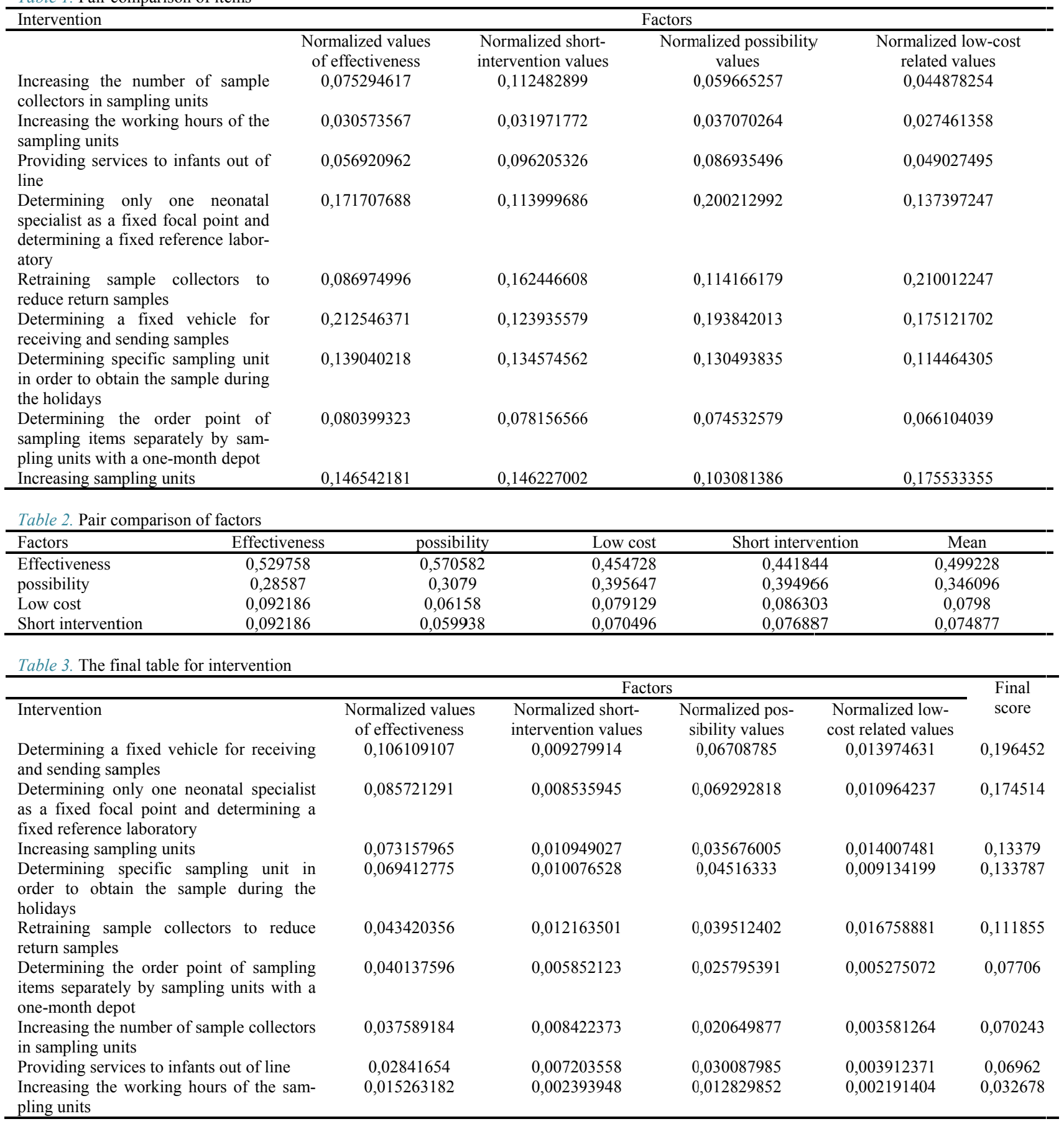


Table 4. Comparison of the time of each one of sampling steps before and after intervention

\begin{tabular}{lccccc}
\hline Time (in days) & Period & Number of samples & Mean & Variance & P value \\
\hline First time sampling & Before model implementation & 3362 & 4.679 & 3.087 & $<0.001$ \\
Second time sampling & After model implementation & 4392 & 4.39 & 2.833 & $<0.001$ \\
& Before model implementation & 197 & 18.969 & 9.796 & $<0.049$ \\
Treatment onset & After model implementation & 182 & 13.522 & 3.59 & \\
& Before model implementation & 11 & 21.72 & 7.72 & \\
\hline
\end{tabular}

Table 5. Comparing the sigma level of the first sampling turn and treatment onset

\begin{tabular}{lccc}
\hline & Period & DPMO & Sigma level \\
\hline First turn of sampling & Before model implementation & 189797 & 2.38 \\
& After model implementation & 175214 & 2.43 \\
Treatment onset & Before model implementation & 181818 & 2.41 \\
& After model implementation & 58824 & 3.06 \\
\hline
\end{tabular}

(before the intervention) to 17.41 days (after the intervention).

Upon implementing the Six Sigma model, the sigma level of the time of the first sampling task and the treatment improved (Table 5).

\section{Discussion}

This study aimed to investigate the effects of the Six Sigma model on reducing the treatment initiation time in patients with congenital hypothyroidism. According to the findings of this study, the implementation of the Six Sigma model reduced the first and second screening time in neonates, thereby resulting in a reduction in the treatment initiation time among patients. This reduction in the treatment time resulted in a higher percentage of patients receiving treatment in the neonatal period.

No similar studies have been conducted to compare the results of this study with those of other studies. However, other interventions have been made to reduce hospitalization and waiting time. The experience of the Dutch Red Cross Hospital showed that Six Sigma could produce significant results in less than 6 months. Three instances of success for this model occurred in the operating room, during the postpartum stay, and after the antibiotic use. The length of stay decreased after the implementation of this model (12). In a study conducted in Jordan entitled 'reducing the waiting time at an emergency department using Six Sigma' at a 273-bed hospital, the implementation of the Six Sigma model decreased the average waiting time and length of stay for $33.21 \pm 15.77$ and $84.49 \pm 26.66$ minutes, respectively. In addition, this study showed that the implementation of this model reduced the length of stay and the waiting time by $34 \%$ and $61 \%$ without adding personnel, respectively. After adopting this approach, the sigma level of the waiting time improved from 0.66 to 5.8 , and that of the length of stay increased from 0.58 to 3.09 (16). Another study was conducted aimed at increasing the quality of trauma care services and improving the discharge method using the Six Sigma model at the Trauma Center of the Groningen University in 2008. In this study, the average length of stay at the beginning of the project was 10.4 days before the intervention, which was reduced to 8.5 days after the intervention. In addition, the average length of stay for all the patients (surgery and trauma) was 2.9 days shorter than that before the intervention. In addi- tion, the average length of stay in trauma patients decreased from 11.8 to 8.5 days (19). Another study showed that intervention in the workflow process reduced the waiting time for triage and scheduled visits (20).

A study conducted to reduce the time of discharge of patients in the emergency dlepartment of the hospital showed that a series of six-sigma interventions within 10 months reduced the average length of stay of patients from 3.4 days to 3.1 days after the intervention. The time of discharge process was reduced from 2.2 hours to 1.7 hours after the intervention, and the length of stay of hospitalized patients in the post-intervention period was reduced from $6.9 \pm 7.8$ hours to $5.9 \pm 7.7$ hours $(21)$.

Such a decrease in time after the intervention indicates the consistency of the results of these studies with those of the present study.

In the present study, the Six Sigma model was used for the first time to reduce treatment delays in neonates with congenital hypothyroidism. In addition to the health units, the present study made the intervention at hospitals, central laboratories, and referral-second-level, i.e., neonatal specialists, involved.

\section{Research limitations}

This study was a case study with its results being probably valid only under conditions of the project research. Therefore, its results should be generalized to other environments inside or outside Iran with caution.

\section{Conclusion}

Six Sigma could be used as an intervention tool for improving indices of health intervention processes. When using this model, enough attention should be paid to the intervention subject and conditions of the intervention environment. In fact, the final decision about the effects of this model in different environments needs conducting further research on this subject.

\section{Ethics approval and consent to participate}

This article is the product of a project approved by the vice president of research at Mashhad University of Medical Sciences (code 940195) under the code of ethics IR.MUMS.REC.1395.321. Although this study was a process study and was not performed on human participants, administrative permissions to access and use the 
medical records described in this study, obtained and approved by Mashhad University of Medical Sciences.

\section{Acknowledgment}

The researcher would like to extend his gratitude to the staff at Samen Health Center for their valuable assistance in this project.

\section{Conflict of Interests}

The authors declare that they have no competing interests.

\section{References}

1. Veisani Y, Sayehmiri K, Rezaeian S, Delpisheh A. Congenital hypothyroidism screening program in iran; a systematic review and metaanalysis. Iran J Pediatr. 2014;24(6):665.

2. Najafian B, Shahverdi E, Afsharpaiman S, Shohrati M, Karimi S, Konjedi MA. Neonatal screening for congenital hypothyroidism in an university hospital in Tehran, Iran (Brief Report). J Compr Ped. 2016;7(2):0-0.

3. Eftekhari N, Asadikaram GR, Khaksari M, Salari Z, Ebrahimzad. The prevalence rate of congenital hypothyroidism in Kerman. Iran in 20052007. J Kerman Univ Med Sci 2015;15:243-250.

4. Büyükgebiz A. Newborn screening for congenital hypothyroidism. J Clin Res Pediatr Endocrinol. 2013;5(Suppl 1):8-12.

5. Glorieux J, Dussault JH, Letarte J, Guyda H, Morissette J. Preliminary results on the mental development of hypothyroid infants detected by the Quebec Screening Program. J Pediatr. 1983 Jan 1;102(1):19-22.

6. Agrawal P, Philip R, Saran S, Gutch M, Razi MS, Agroiya P, et al. Congenital hypothyroidism. Indian $\mathrm{J}$ Endocrinol Metab. 2015;19(2):221.

7. Dhondt JL, Farriaux JP, Sailly JC, Lebrun T. Economic evaluation of cost-benefit ratio of neonatal screening procedure for phenylketonuria and hypothyroidism. J Inherit Metab Dis. 1991;14(4):633-639.

8. Rahmani K, Yarahmadi S, Etemad K, Mehrabi Y, Aghang N, Koosha A, et al. Importance of Early Treatment in Congenital Hypothyroidism. Iran J Pediatr. 2017;27(3):e9438.

9. Rastogi MV, LaFranchi SH. Congenital hypothyroidism. Orphanet J Rare Dis. 2010;5(17).

10. Hughes R. (Ed.). Patient safety and quality: An evidence-based handbook for nurses . (Prepared with support from the Robert Wood Johnson Foundation). AHRQ Publication No. 08-0043. Rockville, MD: Agency for Healthcare Research and Quality; March 2008.

11. Liberatore MJ. Six Sigma in healthcare delivery. Int J Health Care Qual Assur. 2013 Aug;23.

12. Van Den Heuvel J, Does R J, Verver JP. Six Sigma in healthcare: lessons learned from a hospital. Int J Six Sigma Compet Adv. 2005;1(4), 380-388.

13. Pellicone A, Martocci M. Faster turnaround time. Qual Progress. 2006;39(3):31-36.

14. Gayed B, Black S, Daggy J, Munshi IA. Redesigning a joint replacement program using Lean Six Sigma in a Veterans Affairs hospital. JAMA Surg. 2013;148(11):1050-1056.

15. Taner MT, Sezen B, Antony J. An overview of six sigma applications in healthcare industry. Int J Health Care Qual Assur. 2007 Jun; 20(4):329-340.

16. Mandahawi N, Al-Shihabi S, Abdallah AA, Alfarah YM. Reducing waiting time at an emergency department using design for Six Sigma and discrete event simulation. Int J Six Sigma Compet Adv. 2010;6(12):91-104.

17. Lande M, Shrivastava RL, Seth D. Critical success factors for Lean Six Sigma in SMEs (small and medium enterprises). TQM J. 2016;28(4):613-635.

18. Leal JE. AHP-express: A simplified version of the analytical hierarchy process method. MethodsX. 2020;7:100748.

19. Niemeijer GC, Trip A, Ahaus KT, Does RJ, Wendt KW. Quality in trauma care: improving the discharge procedure of patients by means of Lean Six Sigma. J Trauma. 2010;69(3):614-619.

20. Fischman D. Applying Lean Six Sigma methodologies to improve efficiency, timeliness of care, and quality of care in an internal medicine residency clinic. Qual Manag Health Care. 2010;19(3):201-
210.

21. El-Eid GR, Kaddoum R, Tamim H, Hitti, EA. Improving hospital discharge time: a successful implementation of six sigma methodology. Medicine. 2015;94(12). 